Original Article

\title{
A Study in Aerodynamic Optimization of UAV Helicopter Rotor- Blades Planform in Vertical Motion
}

\author{
M F Afthon ${ }^{1,}$, M A Moelyadi ${ }^{1}$ \\ 1 Faculty of Mechanical and Aerospace Engineering, Institut Teknologi Bandung, Indonesia. \\ * Correspondence: afthonm66@gmail.com
}

Received: 12 January 2020; Accepted: 30 April 2020; Published: 30 June 2020

\begin{abstract}
According to the objectivity of UAV helicopter, endurance is a valuable performance. To increase the endurance, we need to decrease the helicopter required power. Within the research scope in vertical movement only, 5 parameters of blades planform design were considered as design variables. They are root chord of the blades, taper location, taper ratio, pitch angle, and tip twist angle. Optimization was done using own developed genetic algorithm codes with built-in blade element momentum theory (BEMT) as a performance calculator. It was chosen due to its ability to estimate rotor performance quickly. Several CFD simulation were done to reduce the error of blade element momentum theory calculation. Using constant adjustment methods, BEMT can predict thrust and power with a difference with respect to CFD of $3.8 \%$ and $8.2 \%$ respectively. The optimization result gives the optimum blades design with improving almost $11 \%$ in efficiency which came out from $9.4 \%$ reduction in power required which is good for helicopter performance.
\end{abstract}

Keywords: aerodynamic performance, optimization, genetic algorithm, rotor design.

\section{Introduction}

In preliminary design step, the best planform of the rotor blades must be chosen according to optimize the rotor performance [1]. Commonly, researchers optimize the performance of rotor by selecting the best shape of the blade (airfoil shape and blade planform) using integrated analytical programs or numerical like CFD. In this study, analytical calculation was chosen due to the need of quick optimization in preliminary design phase [2].

The air flew through the rotor-blades generate lift and drag, which results thrust and torque acting on the rotor [3]. Performance of the rotor mostly defined by three quantities, i.e. thrust, torque or power required, and aerodynamic efficiency. It is convenient to express thrust and power required in terms of non-dimensional quantities so called thrust coefficient and power coefficient which expressed as follows.

$$
\begin{aligned}
& C_{T}=\frac{T}{\rho \Omega^{2} D^{4}} \\
& C_{P}=\frac{P}{\rho \Omega^{3} D^{5}}
\end{aligned}
$$

Where, $T$ is thrust produced by the rotor, $P$ is power required, $\rho$ is air density, $\Omega$ is the rotational speed of the rotor, and $D$ is the rotor diameter. While the rotor aerodynamic efficiency can be written as follows.

$$
\begin{gathered}
\eta=\frac{C_{T}}{C_{P}} J \\
J=\frac{V}{n D}
\end{gathered}
$$


Where $J$ is called as advance ratio which describe the ratio between axial and rotational speed of the airflow.

The aim of this optimization is to minimize the rotor power required at a specified thrust level in vertical movement of the helicopter. Specified thrust must be equal or higher than MTOW of the helicopter to make it hover, at least. Reducing the power required by the rotor can save more energy thus will increase its endurance.

To optimize helicopter rotor performance, it needs to define its design variables. Mostly, variables which contribute greatly on blade performance are its airfoil, chord, and twist distribution. In this study, airfoil is set to be constantly distributed along the radius. Thus, only chord and twist distribution become the design variables. 5 parameters are defined to express the chord and twist distributions.

In order to do a cross-validation with CFD, dummy datum model was constructed by replicating Alva800 helicopter blade. Its sizing and main geometrical data also become the baseline. Datum model was analysed using CFD to calculate the rotor thrust and power [3].

Before starts the optimization process, 20 samples were being analysed using CFD and blade element- momentum theory (BEMT). Average difference in thrust, power required, also both standard deviations become the correction to the BEMT program. The optimization process started by defining 400 samples with varying those 5 variables. Halton sequence method is used in this sampling process due to its excellence to spread the samples evenly in the design space [4]. Then, BEMT program calculate performance of those samples.

The results were inputted to the genetic algorithm program to do the iterative single objective optimization until it reaches the iteration limit. Schematic of this research process is appeared at Figure 1. After got the optimization results, the blade model which has 5 optimized parameters was analysed using CFD to build a rotor efficiency diagram and compare it with the datum. Optimization program runs 3 times with difference setting and the results were compared.

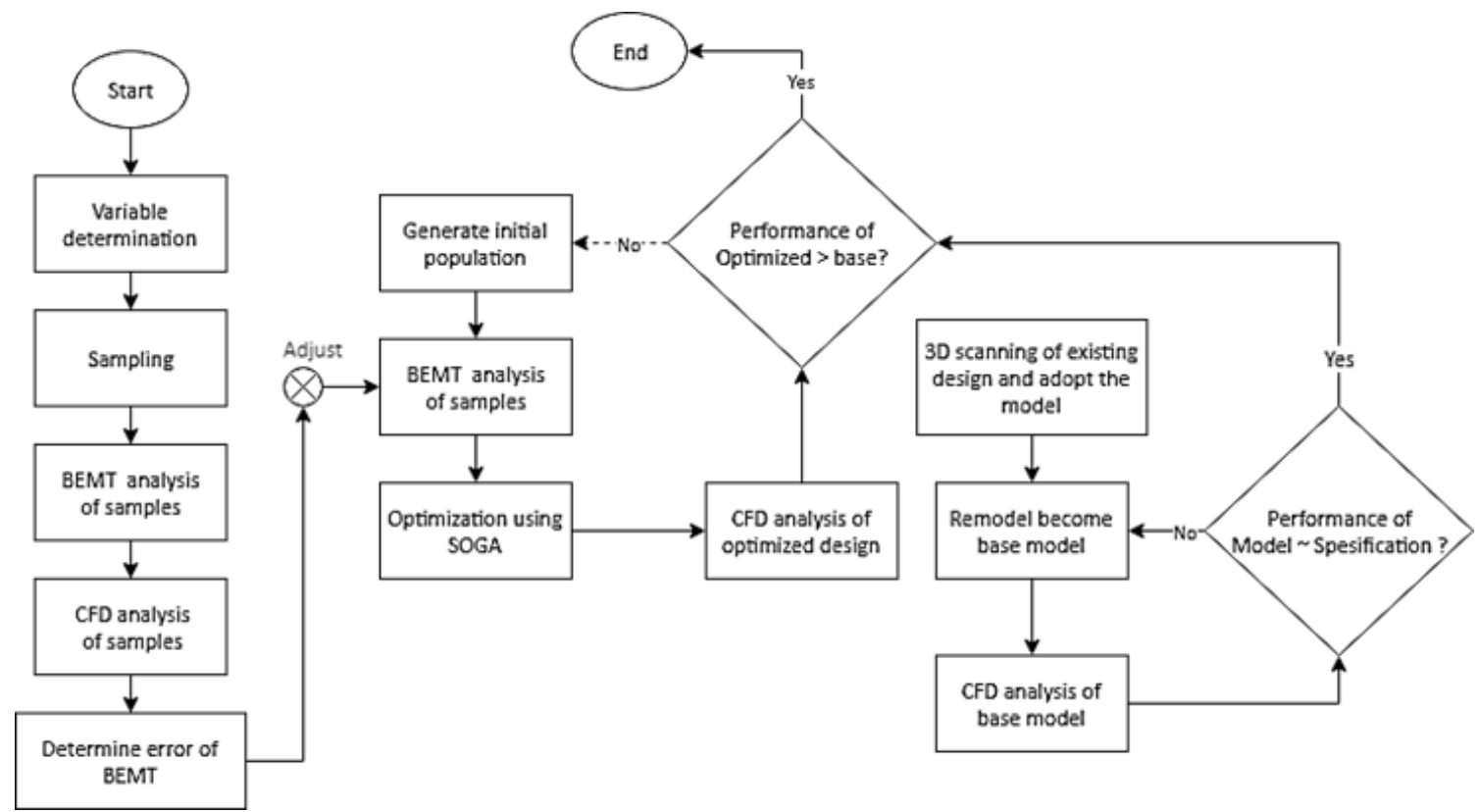

Figure 1. Research schematic. 

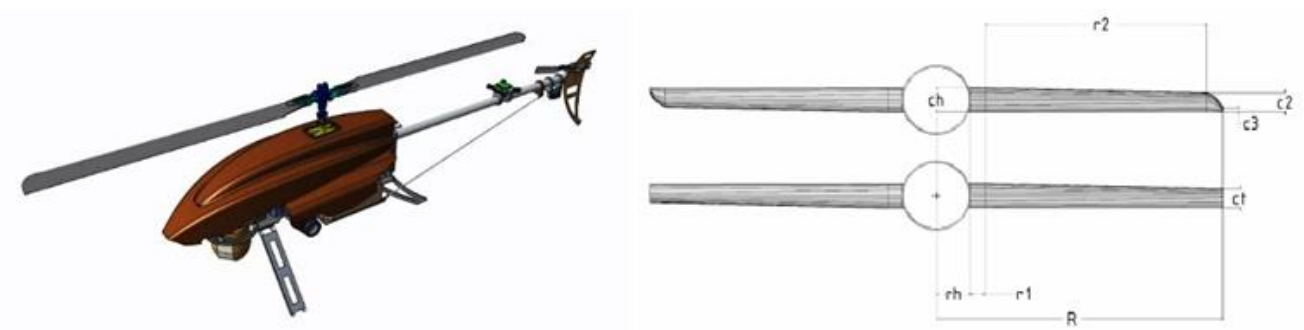

Figure 2. Alva800 model (left), datum \& base rotor model.

\section{Design Consideration}

\subsection{Design objective}

The thrust and power required of the datum model which obtained from CFD analysis becomes the limitation of the optimization process [4]. Thus, the requirements of this design process can be stated as follows, "Optimized blade design must produce thrust the same or higher than MTOW of the helicopter and require a lower power than the datum model required."

\subsection{Datum model and base model}

Datum model is needed due to comparison of the optimization result. This model is adopted from the geometry and sizing of unmanned helicopter Alva800 [5]. The look of datum model and its main specification is shown in Figure 2 and Table 1.

Table 1. Alva800 general data.

\begin{tabular}{cc}
\hline Characteristic & Detail \\
\hline MTOW & $14 \mathrm{~kg}$ \\
Engine & 2-strokes gasoline \\
Vertical speed & $5 \mathrm{~m} / \mathrm{s}$ \\
Rotor geometry & 2 blades, 1.8 diameter \\
RPM & 1400 \\
\hline
\end{tabular}

The blade design of the datum model can be seen in Figure 2. While Table 2 shows all the parameter which can be defined from the datum model. Due to its complexity to be remodeled, then the chord and twist distribution are redefined with only 5 parameters. The simplified model is named as base model. Simplification focusses on neglecting the curved end of the blade-tip. The base model also being analyzed in CFD to calculate its thrust and power required. Base model is needed due to the want to compare analytic optimized design "apple to apple" with CFD result. Design parameter of the base model can be found on Table 3. In this optimization, operation condition from Table 1 to Table 3 shows an equivalent with advance ratio of 0.12 then be the on-design condition.

Table 2. Datum \& base model design parameters.

\begin{tabular}{lccc}
\hline \multicolumn{1}{c}{ Variable } & Sym. & Datum & Base \\
\hline Rotor Radius (mm) & $R$ & 900 & 900 \\
Hub Radius (mm) & $r_{h}$ & 108 & 108 \\
Taper Loc. (mm) & $r_{1}$ & 152 & 152 \\
Breakpoint Loc. (mm) & $r_{2}$ & 697 & - \\
Root Chord (mm) & $c_{h}$ & 77 & 77 \\
Break Loc. chord (mm) & $c_{2}$ & 60 & - \\
Tip Chord (mm) & $c_{t}$ & 12 & 54 \\
Taper Ratio & $t$ & 0.78 & 0.779 \\
Pitch Angle (deg) & $\beta_{h}$ & 8.0 & 8.0 \\
Tip Twist Angle (deg) & $\theta_{t}$ & -2.5 & -2.5 \\
\hline
\end{tabular}


Table 3. Design variable constraints.

\begin{tabular}{|c|c|c|c|c|}
\hline Variable & Sym. & Base & $\begin{array}{l}\text { Lower } \\
\text { Bound }\end{array}$ & $\begin{array}{l}\text { Upper } \\
\text { Bound }\end{array}$ \\
\hline Root Chord (Radius) & $c_{h}$ & 0.086 & 0.05 & 0.3 \\
\hline $\begin{array}{l}\text { Taper Location } \\
\text { (Radius) }\end{array}$ & $r_{1}$ & 0.169 & -0.12 & 1 \\
\hline Taper Ratio & $t$ & 0.779 & 0.2 & 1 \\
\hline Pitch Angle (deg) & $\beta_{h}$ & 8 & 0 & 12 \\
\hline Tip Twist Angle (deg) & $\theta_{t}$ & -2.5 & -12 & 0 \\
\hline
\end{tabular}

\subsection{Design variables and bounds}

Through the optimization process, 5 variables are used to define the chord and twist distribution. They are written in Table 3 . Those 5 parameters are chosen to be design variables due to theirs great effect on rotor performance $[2,6]$.

\subsection{Objective function} follows.

Due to the objectivity of the optimization process stated, objective function can be defined as

$$
f=\frac{\eta}{10}\left(1+9 S_{C T} S_{C P}\left(1-d_{C T}\right)\left(1-d_{C P}\right)\right)
$$

Where $\eta$ is the rotor aerodynamic efficiency. SCT and SCP are the control coefficient which has values 1 when the condition is acceptable and 0 when it's not. 1 and 9 -multiplier is only a weighting factor.

$$
\begin{gathered}
S_{C T}=\begin{array}{l}
1, \text { when } T \geq T_{\text {base }} \\
0, \text { when } T<T_{\text {base }}
\end{array} \\
S_{C P}=\begin{array}{l}
1, \text { when } P \leq P_{\text {base }} \\
0, \text { when } P>P_{\text {base }}
\end{array}
\end{gathered}
$$

$d C T$ and $d C P$ are the correction factor. $d C T$ explains how different the thrust produced by the rotor with the base model. While $d C P$ explains as well as $d C T$ but in terms of power required.

$$
\begin{gathered}
d_{C T}=\frac{C_{T}-C_{T_{\min }}}{C_{T_{\min }}}=\frac{C_{T}}{C_{T_{\text {min }}}}-1 \\
d_{C P}=\frac{C_{P}-C_{P_{\max }}}{C_{P_{\max }}}=\frac{C_{P}}{C_{P_{\max }}}-1
\end{gathered}
$$

Consider a sample rotor produce a higher thrust with a lower power required with respect to the base model CFD result. Thus, $S C T$ and $S C P$ has value of 1 . Then $d C T$ must be a positive number while $d C P$ is negative. When $d C P$ is negative, it is good because it increases the value of the function $f$. But, when $d C T$ is positive, it turns a lower value of $f$. It is set to be like that because the need of rotor that produce sufficient thrust with the lowest power required as possible. As we know that power required increases when thrust increased as well as induced drag rises due to lift. 


\section{Design and Optimization Tools}

\subsection{Blade performance calculator}

Blade element-momentum theory can be used to calculate performance of the rotor. Lift and drag equation from blade element theory is combined with the momentum equation to become the BEMT master equation. Some corrections are imposed in the BEMT master equation to model the hub-tip loss for the blade [7]. Iterative calculation is done until converged. Total lift and drag then can be projected as thrust and torque- force. This program needs aerodynamic data of the airfoil. Thus, airfoil database must be inserted first. Including the data of $\mathrm{cl}$ and $\mathrm{cd}$ with the function of Mach number and Reynolds number. Airfoil database is constructed using common software JavaFoil. This software using high order panel method to calculate velocity distribution due to inviscid velocity. While integral method of boundary layer analysis is used to calculate drag of airfoil [7].

\subsection{Optimization tools}

Genetic algorithm is widely used in aerospace domain. Its simplicity and accuracy in giving optimization solution is the strength. Many developments of this algorithm used to improve its performance for example the NSGA and NSGA-II which can result a set of solutions. GA can perform either single or multiple objectives. Due to the relation between thrust and torque of the rotor which can represented by the rotor efficiency, single objective GA can perform well [8].

\subsection{CFD analysis}

In order to make a cross-validation between analytical and numerical method, CFD simulation is done to analyze datum and base model, also for 20 adjustment samples. Unstructured mesh is used due to the complexity of the blade geometry. Prism mesh is applied near the blade to catch boundary layer phenomena. SST is applied to model the turbulence. Simulation is done using ANSYS with frozen rotor method. CFD simulation result of the datum and base model is appeared in Table 4. The table shows that the simplification of the geometry from datum model become base model does not give a huge difference. Thus, the simplification can be safely applied

Table 4. CFD result: datum vs base.

\begin{tabular}{cccc}
\hline Performance & Datum & Base & Error (\%) \\
\hline Thrust $(\mathrm{N})$ & 147 & 152 & 3.4 \\
Power $(\mathrm{W})$ & 2845 & 2856 & 0.4 \\
\hline
\end{tabular}

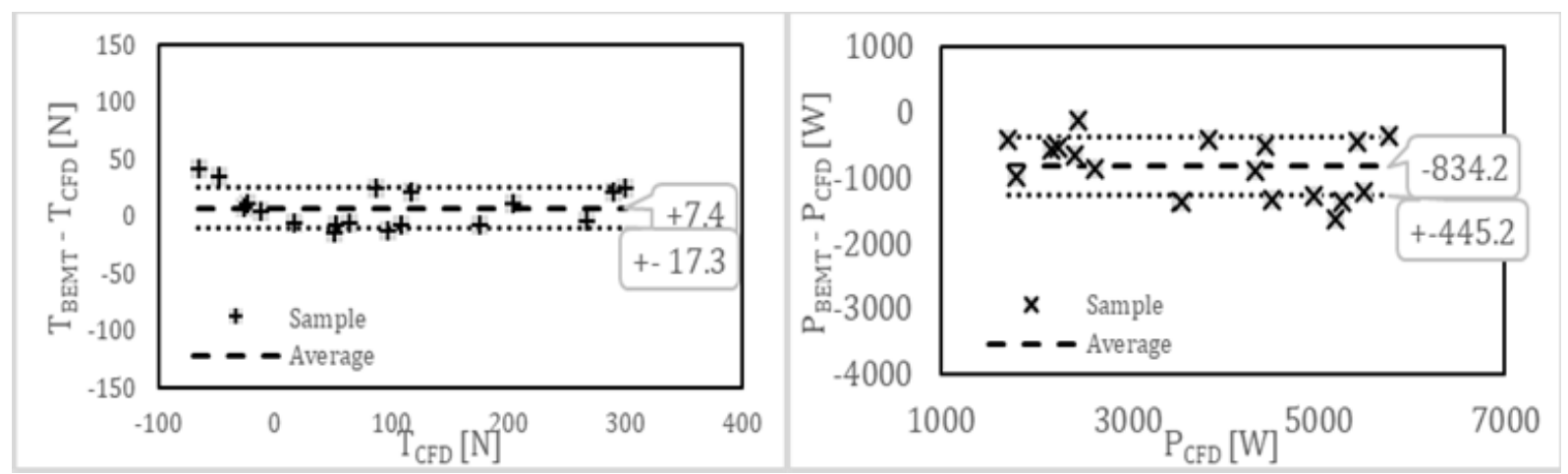

Figure 3. BEMT vs CFD. 


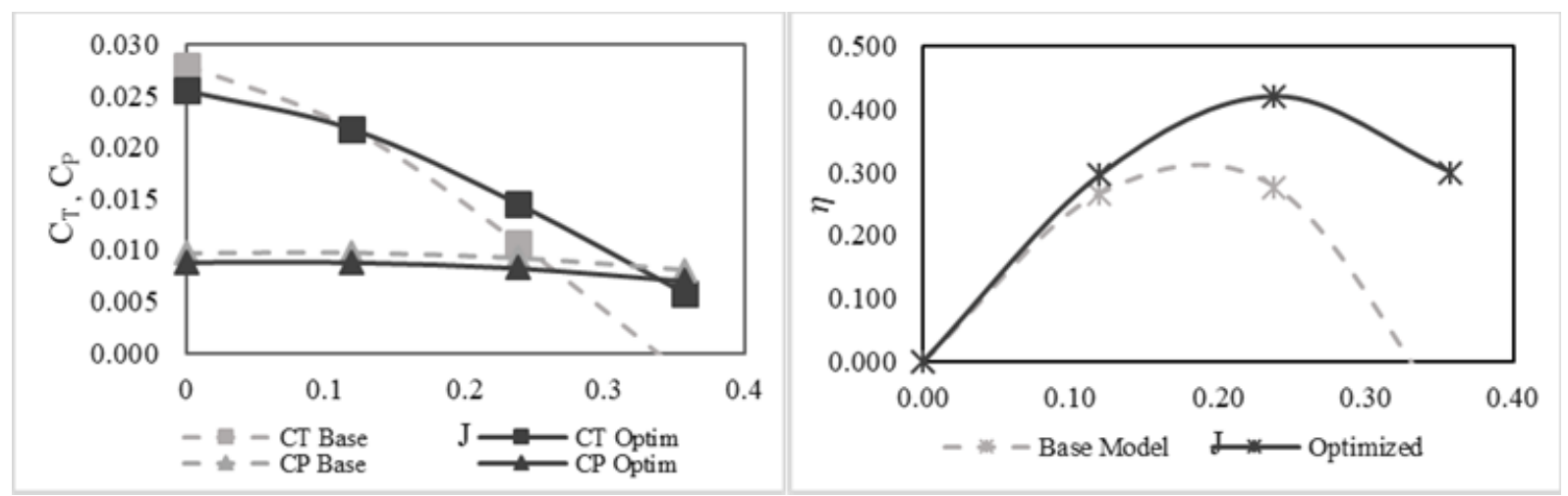

Figure 4. Off-design analysis of optimized-design in various advance ratio J.
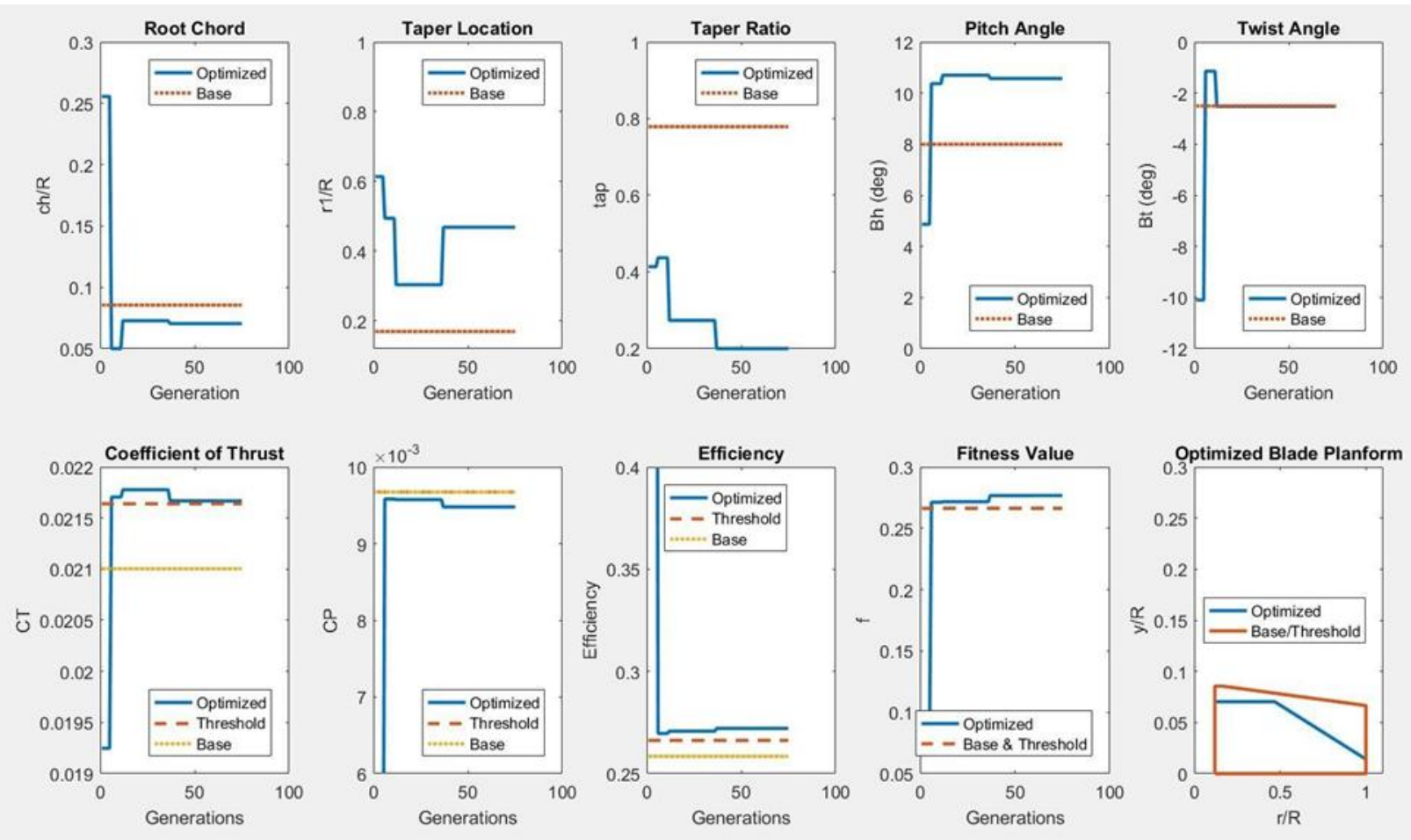

Figure 5. Best design through generation: variables (5 top), performance (4 bottom), the optimized design

The optimization objective has met the requirements that's the rotor can produce the same with the base model but requires lower power. Optimized planform design is founded and appear in Figure 5, as can be compared to other results $[7,8]$.

\subsection{BEMT adjustment}

BEMT is analytical approach to estimate rotor performance which impose many assumptions. Thus, it produces higher error than numerical method (CFD). 20 random rotor design samples are selected to be investigated. Each sample is analyzed using both BEMT and CFD. Then the results are compared in terms of the rotor thrust and power required. Figure 3 shows how BEMT result differ with respect to CFD. it shows that BEMT result is tends to give systematic error which has average difference of $\triangle T a v g$ and $\triangle P a v g$ with respect to CFD simulation.

Also, according to Figure 3 we can safely say that BEMT calculation can be adjusted constantly in the future calculation to get closer with the result of CFD simulation. Thus, we can state the difference between BEMT and CFD performance calculation as follows. 


$$
\Delta=\Delta_{\text {avg }} \pm \sigma
$$

Where $\Delta a v g$ is the average difference while $\sigma$ is the uncertainty which is represented by standard deviation. That's why for performance calculation in the whole optimization algorithm will use BEMT with imposing this correction.

$$
(T, P)_{\text {adjusted }}=(T, P)_{B E M T}-(\Delta T, \Delta P)_{\text {avg }}
$$

While the uncertainty $\sigma \Delta$ is used for thresholding so that the requirements can be stated as follow.

$$
\begin{array}{ll}
T_{\text {min }}=T_{\text {base }}+k \sigma_{T} & P_{\text {max }}=P_{\text {base }}-k \sigma_{P} \\
T_{\text {min }}=T_{\text {base }}+k \sigma_{T} & P_{\text {max }}=P_{\text {base }}-k \sigma_{P}
\end{array}
$$

Where $k$ is an arbitrary coefficient which is obtained by trial and error.

\section{Results and Discussions}

According to Figure 3 and Table 5, it's effective using BEMT adjustment to reduce the error of the program correspond to the CFD simulation. Figure 5 shows how the calculated performance of the best design in population has changed through generations. Performance of the rotor is shown in 4 terms i.e. thrust coefficient, power coefficient, efficiency, and fitness function values $[7,8]$. The figure also shows the plot of 5 design variables correspond to the best design in population through generations. Table 6 provides the optimized rotor planform design and the improvements. Off-design CFD analysis of the optimized-design is shown in Figure 4. It shows that the optimized design gives better efficiency in all advance ratio.

Table 5. Difference of BEMT and CFD after imposing correction.

\begin{tabular}{cccccccccc}
\hline & \multicolumn{3}{c}{ BEMT } & \multicolumn{3}{c}{ CFD } & \multicolumn{3}{c}{ Difference (\%) } \\
\cline { 2 - 11 } Case & $\boldsymbol{T}[\boldsymbol{N}]$ & $\boldsymbol{P}[\boldsymbol{W}]$ & $\boldsymbol{\eta}$ & $\boldsymbol{T}[\boldsymbol{N}]$ & $\boldsymbol{P}[\boldsymbol{W}]$ & $\boldsymbol{\eta}$ & $\boldsymbol{\Delta T}$ & $\boldsymbol{\Delta P}$ & $\boldsymbol{\Delta} \boldsymbol{\eta}$ \\
\hline 1 & 147.2 & 2628.5 & 0.280 & 164.8 & 3007.2 & 0.274 & 10.7 & 12.6 & 2.2 \\
2 & 147.1 & 2679.3 & 0.274 & 146.8 & 2571.5 & 0.285 & 0.2 & 4.2 & 3.8 \\
3 & 151.7 & 2787.4 & 0.272 & 152.6 & 2587.0 & 0.295 & 0.6 & 7.7 & 7.7 \\
\hline \multicolumn{4}{c}{ Average Difference } & & 3.8 & 8.2 & 4.6 \\
\hline
\end{tabular}

Table 6. Optimized-design review.

\begin{tabular}{ccccc}
\hline Design Variable & Sym. & Base & Optimized & Improvement \\
\hline Root Chord [R] & $c_{h}$ & 0.086 & 0.070 & \\
Taper location [R] & $r_{1}$ & 0.169 & 0.469 & \\
Taper ratio & $t$ & 0.779 & 0.200 & \\
Pitch Angle (deg) & $\beta_{h}$ & 7.00 & 10.57 & \\
Tip Twist (deg) & $\theta_{t}$ & -2.50 & -2.52 & \\
Thrust Produced & $T$ & 152.0 & 152.6 & $0.34 \%$ \\
Power Required [W] & $P$ & 2856 & 2587 & $-9.41 \%$ \\
Efficiency & $\eta$ & 0.266 & 0.295 & $10.77 \%$ \\
& & & & \\
\hline
\end{tabular}




\section{Conclusions}

From this study we also can interpret that analytical calculation of rotor performance is tends to make a systematic error than a random error. Thus, for a specified flight condition, constant adjustment can perform well to reduce the inaccuracy of the calculation. In this case, BEMT program with adjustment can predict thrust and power required of the rotor with difference respect to CFD of $3.8 \%$ and $8.2 \%$ respectively. The optimized design increases rotor efficiency in on-design operation by almost $11 \%$ which came out from $9.4 \%$ power reducing. While, in off-design operation, the optimized-design provides higher efficiency than the base model in all advance ratio, which is good for rotor optimization with the aim of rising its endurance.

\section{References}

1. Walsh, Bingham and Riley, "Optimization method applied to aerodynamic design of helicopter rotor blades," J Am Helicopter Soc, vol. 32(4), pp. 39-44, 1987.

2. N. A. Vu, J. W. Lee and J. I. Shu, "Aerodynamic design optimization of helicopter rotor blades including airfoil shape for hover performance," Chinese Journal of Aeronautics, vol. 26(1), pp. 1-8, 2013.

3. T. J. Lowry, Performance of Light Aircraft, Billings, Montana: American Institute of Aeronautics and Astronautics, Inc, 1999.

4. Wikipedia Foundation, Inc, "Halton Sequence," $26 \quad 9$ 2017. [Online]. Available: https://en.wikipedia.org/wiki/Halton_sequence. [Accessed 15 2018].

5. Bhimasena Research \& Development, "Spesifikasi Teknik "OPTERA"," Bhimasena Research \& Development, Bandung, 2017.

6. Leishman JG, Principles of helicopter aerodynamics, Cambridge: Cambridge University Press, 2006.

7. W. Z. Shen, R. Mikkelsen, J. N. Sorensen and C. Bak, "Tip loss corrections for wind turbine computations," Wind Energy 8, pp. 457-475, 2005.

8. Hepperle, "Aerodynamics of Model Aircraft," 2008. [Online]. Available: https://www.mhaerotools.de/airfoils/index.htm.

This is an open-access article distributed under the terms of the Creative Commons Attribution 4.0 International License, which permits unrestricted use, distribution, and reproduction in any medium provided the original work is properly cited. 